\title{
Imitation Assessment and Its Utility to the Diagnosis of Autism: Evidence from Consecutive Clinical Preschool Referrals for Suspected Autism
}

Marleen Vanvuchelen · Herbert Roeyers · Willy De Weerdt

\begin{abstract}
The present study sought to examine imitation difficulties as a risk factor for autism. Imitation aptitude was examined in 86 preschoolers suspected of autism (1.9-4.5 years) using the Preschool Imitation and Praxis Scale (PIPS). Differences between imitation, language, motor age-equivalents and nonverbal mental age were used to predict the diagnosis of autism. Multidisciplinary team diagnoses and ADOS-G classifications were used to differentiate children with autism spectrum disorders and non-spectrum developmental disorders. Two factors were found to be significantly associated with autism using simple logistic regression analyses: procedural imitation delay and receptive language delay. In a multivariable setting, only procedural imitation delay remained a significant predictor of autism. Results are new to the literature and require replications.
\end{abstract}

Keywords Bodily and procedural imitation - Assessment - Core deficit - Differential diagnosis - Preschool Imitation and Praxis Scale (PIPS) - Cohort type diagnostic accuracy study 
Autistic disorder and related autism spectrum disorders are neurodevelopmental disorders characterised by impairment in social interaction, in communication skills and in behaviour, which is restricted and repetitive (American Psychiatric Association 2000). In this paper the term Autism Spectrum Disorders (hereafter 'autism') encompasses Autistic Disorder, Asperger's Disorder and Pervasive Developmental Disorder-Not Otherwise Specified. Growing awareness of symptoms of autism in preschoolers among parents and professionals results in a rapidly increasing number of young children being referred to specialised clinics for a differential diagnosis. There is growing evidence that diagnosis of autism by age 3 , and even by age 2, is stable over time (Chawarska et al. 2007; Kleinman et al. 2008). However, the median age of identification is 5.7 years (Shattuck et al. 2009). In a cohort of children younger than 12 years of age in Flanders, the Dutch-speaking region of Belgium, the average age of autism diagnosis was 5.9 years (Roeyers 2008). The long delay between parents' initial concerns and eventual diagnosis postpones appropriate intervention, which leaves parents with the sense that precious time has been lost (Wiggins et al. 2006). Autism diagnosis in young children may be delayed due to doubts about diagnostic validity. This may be due to several factors, including the fact that symptom presentation in autism varies over time. One method to increase diagnostic validity may be the application of specific instruments to assess the age-related syndrome expression of autism (Charman and Baird 2002). On the other hand, it is not evident to isolate a single symptom from the heterogeneous picture of social and nonsocial characteristics seen in autism (Southgate and Hamilton 2008).

The present study focuses on the contribution of imitation assessment to the diagnosis of autism at preschool age. An important issue has to be addressed prior to the application of an imitation instrument in the diagnostic protocol for autism. Do imitation problems reflect a core characteristic in autism? A symptom is considered to be a core characteristic of autism if it is unique to autism, specific and universal (Sigman et al. 2004). In addition, a deficit must fulfil the criteria of persistency (Hobson and Lee 1999), precedence (Rogers 1999) and broadness.

There is some evidence that imitation problems in infancy, together with several other indicators, proceed and predict the diagnosis of autism (Zwaigenbaum et al. 2005). Furthermore, findings of subtle imitation problems in adolescents and adults with autism support the criterion of persistency (Hobson and Lee 1999). Up till now, the requirement that imitation problems are unique to autism has led to case-control studies that compared 
imitation aptitude of groups of children with autism to control groups of typically developing children or children with different disabilities, matched for age and level of development. In a comprehensive review Williams et al. (2004) pooled the findings from twelve well-controlled case-control studies, involving 196 individuals with autism. They calculated the combined $p$ value of group differences with respect to imitation problems to an appropriate control group, resulting in a $p$-value of 0.00002 . This finding supports the criterion of uniqueness of a core deficit in autism (Williams et al. 2004). To establish the specificity of imitation problems, research is carried out that contrasts different functions and abilities across groups, testing the hypothesis that imitation problems are deficient in the autism group while other problems are not involved, or that imitation problems are specific rather than being part of a more general problem (Sigman et al. 2004). Results of Williams and colleagues' meta-analysis revealed that mental delay and motor skill impairment account for some impairment but by no means for all of it. This finding supports the criterion of specificity of a core deficit in autism (Williams et al. 2004).

Less evidence is found for the idea that imitation problems are universal and broad in autism. For the criterion of universality to be met, a core deficit is expected to appear during at least one age period in all individuals with autism, regardless of the severity of the disorder (Sigman et al. 2004). Since in Williams and colleagues' meta-analysis the size of the imitative problem was most apparent in younger age groups, it seems a valuable idea to explore this criterion at preschool age, including children with different functional levels.

The requirement that the deficit is broad has led to studies that investigate bodily imitation, i.e., imitation of gestural and facial actions, and procedural imitation, i.e., imitation of actions with objects. The question if preschool children with autism do display a broad or a selective imitation problem remains subject of debate. The majority of studies asked preschoolers to copy bodily actions. These studies, which tap the full range of functional levels, reported consistently bodily imitation problems in the children with autism (Williams et al. 2004; Rogers and Williams 2006). In contrast to bodily imitation, research on procedural imitation is less conclusive, varying from robust (Charman et al. 1997) to no procedural imitation problems in preschoolers with autism (Ingersoll et al. 2003; McDonough et al. 1997; Rogers et al. 2008). In fact, only studies that investigate both bodily and procedural imitation in the same preschoolers with autism compared to appropriate controls have the potential to unravel this question. Two studies found neither bodily nor procedural imitation problems in 
preschoolers with autism, because their results were confounded by ceiling effects (BeadleBrown and Whiten 2004; Carpenter et al. 2002). Most studies found that mentally impaired preschoolers with autism performed significantly poorer during bodily and procedural imitation than developmentally delayed controls (DeMeyer et al. 1972; Roeyers et al. 1998; Stone et al. 1990, 1997) and typically developing children (Stone et al. 1990, 1997). In three studies group differences on gestural imitation were of a greater magnitude than these on procedural imitation (DeMeyer et al. 1972; Roeyers et al. 1998; Stone et al. 1997). The research group of Aldridge et al. (2000) concluded that mentally impaired preschoolers with autism were poorer on bodily imitation, but better on procedural imitation than mental age matched typically developing infants (Aldridge et al. 2000). Contrarily, Rogers et al. (2003) found in toddlers with autism, compared to both typically developing and developmentally delayed peers, impairments in facial and procedural imitation, but not in gestural imitation (Rogers et al. 2003).

The diagnostic utility of a specific instrument to determine whether or not a patient has a target condition should be investigated in a random sample of the indicated population, i.e., patients suspected of the target condition (Jaeschke et al. 1994). The quality of an instrument to distinguish severely affected children, as children with autism are, from healthy children, tells us nothing about the clinical utility of the instrument. In addition, case-control study designs which compare the target condition with people who have clearly other disorders, overestimate the diagnostic utility of an instrument (Jaeschke et al. 1994).

This review revealed that imitation studies in preschoolers with autism were all case-control studies. Therefore imitation problems may be inflated. Up till now, studies have only investigated low-functioning children with autism. The question if imitation problems are universal to all preschoolers with autism remains unanswered. In these studies, tasks have been used that do not indisputably assess imitative behaviour. Some of the bodily imitation tasks were so simple that they barely seemed to tap imitative phenomena (Rogers et al. 2003). In addition, the presence of the objects could potentially evoke a number of non-imitative behaviours, such as replication of skilled acts (Aldridge et al. 2000; DeMeyer et al. 1972; Stone et al. 1990). All studies have used imitation tests without normative data. It is perhaps not surprising then, that some studies were confounded by ceiling effects. They have used a narrow range of tasks, which does not allow the investigation of underlying mechanisms of imitation in this population. Finally, these studies have used chronological age as a criterion 
to match mentally impaired children with autism to typically developing controls (Stone et al. $\underline{1990}$ ), which does not allow ruling out mental problems as the cause of imitation problems.

The present study sought to address the question if imitation problems at preschool age are broad, specific, universal and unique to autism, using a cohort of consecutive clinical preschool referrals for suspected autism. Imitation aptitude was investigated by means of the Preschool Imitation and Praxis Scale (PIPS). The PIPS is designed to assess the accuracy of bodily and procedural imitation performance in children between 1 and 4.9 years of age (Vanvuchelen 2009; Vanvuchelen et al. 2010). Since autism occurs along a broad continuum of mental and language capacities, nonverbal mental age was used as reference criterion. Imitation delay was defined as an imitation development below the child's nonverbal mental age expectancy. There are five questions to be answered: Are imitation problems broad or selective in preschoolers with autism? We hypothesised that preschoolers with autism will have broad imitation problems. Their ability to copy bodily as well as procedural imitation tasks will be below their nonverbal mental age expectancy. Are imitation problems specific instead of being part of other developmental problems? We hypothesised that in preschoolers with autism imitation performance can be partially explained by motor problems. Are imitation problems universal in autism? We hypothesised that all preschoolers with autism will have imitation problems. Are imitation problems unique to autism at preschool age? We hypothesised that preschoolers who have been suspected of autism and who did not meet the criteria of autism will not have imitation problems. We assumed that their imitation performance will be in accordance with their nonverbal mental abilities. What is the diagnostic utility of imitation assessment in preschoolers suspected of autism? To address this question, we investigated the value of bodily and procedural imitation delay as single factors and in conjunction with receptive and expressive language, gross and fine motor delay to predict the diagnosis of autism spectrum disorders in contrast to non-spectrum developmental disorders. 


\section{Methods}

\section{Participants}

Eighty-six preschoolers (24 female and 62 male) suspected of autism and consecutively referred to University Autism Clinics were included in this study. The participants were free from any medical condition and had no visual or hearing impairment. They ranged between 1.9 and 4.5 years of age with a mean chronological age (CA) of 41.5 months, SD 8.4 months. They were diagnosed according to a multidisciplinary clinical consensus classification and compromised two groups: 68 children with a positive diagnosis (ASD, autism spectrum disorders; 18 female and 50 male; $\mathrm{CA}=40.8 \mathrm{~m}$, SD $8.4 \mathrm{~m}$; nonverbal mental age $\mathrm{NMA}=36.6 \mathrm{~m}, \mathrm{SD} 12.6 \mathrm{~m}$ ) and 18 children with a negative diagnosis (NS-DD, nonspectrum developmental disorders; 6 female and 12 male; $\mathrm{CA}=44.3 \mathrm{~m}, \mathrm{SD} 8.3 \mathrm{~m}$; $\mathrm{NMA}=36.8 \mathrm{~m}, \mathrm{SD} 9.1 \mathrm{~m})$.

The NS-DD sample was a heterogeneous group. A clinical diagnosis of mixed receptiveexpressive language disorder was given to seven participants of whom one received an additional diagnosis of mild mental retardation; one of Developmental Coordination Disorders (DCD) and one of Attention-Deficit Hyperactivity Disorder (ADHD) according to DSM-IV-TR criteria (American Psychiatric Association 2000). The diagnosis of expressive language disorder was given to three participants of whom one received an additional diagnosis of DCD. The diagnosis of receptive language disorder combined with DCD was given to one participant. Two participants received a clinical diagnosis of DCD and three participants of mental retardation. Two participants in the NS-DD group did not receive any diagnosis.

This study was approved by the ethics committees of the University Hospitals Louvain, Antwerp, Brussels and Ghent (Flanders, Belgium) before the collection of data. All families gave written informed consent for the participation of their child. 


\section{Measures}

\section{Multidisciplinary Clinical Consensus Diagnosis}

A team consisting of child psychiatrists, paediatricians, psychologists, speech therapists, physical therapists in four by the Belgian Government certified University Autism Clinics observed the children in different contexts according to DSM-IV-TR diagnostic criteria for autism (American Psychiatric Association 2000). Parents and caregivers provided detailed information on developmental history, and everyday behaviour and activities of the child. When a case was considered difficult to assess, clinical consensus classification was reached through reviewing and discussing the available information and observation reports.

\section{Autism Diagnostic Observation Schedule-Generic (ADOS-G) (Lord et al. 2003)}

The ADOS-G is a semi structured, play based assessment which provides systematic probes for autism symptoms in social interaction, communication, play, and repetitive behaviours and interests. The ADOS-G was administered by trained investigators. Module 1 (minimal to no language) or module 2 (non-echoed phrase speech) was used according to the expressive language level of each child. In the group with a clinical diagnosis of ASD, 35 participants received module 1 (mean total score $=10.8$, SD 3.5) and 33 participants module 2 (mean total score $=11.9$, SD 4.2). In the group with a clinical diagnosis of NS-DD, 5 participants received module 1 (mean total score $=3.8, \mathrm{SD} 2.4$ ) and 13 participants module 2 (mean total score $=1.9$, SD 1.4). The clinical diagnosis was confirmed by the ADOS-G-classification in $92 \%$ of the participants. The ADOS-G classification was negative in six children with a clinical diagnosis of ASD and positive in one child with a clinical diagnosis of NS-DD. The ADOS-G provides algorithm criteria for classification of autism and ASD separately: 30/62 (48\%) met the criteria for autism and 32/62 (52\%) for ASD. In the present study, autism and ASD were considered as one group. The mean time between imitation assessment and ADOS administration was 0.15 month (SD 1.0 month).

\section{Preschool Imitation and Praxis Scale (PIPS) (Vanvuchelen 2009)}

The PIPS is a multidimensional imitation test to investigate bodily (gestural and facial) and procedural imitation in children between 12 and 59 months of age. To construct the PIPS, 
action types with different effects (salient environmental and internal), representational levels (meaningful, and non-meaningful; goal directed and non-goal directed), temporal complexities (single and sequential) and visual monitoring possibilities (transparent and opaque) were chosen to tap the full range of possible imitation mechanisms. Imitation tasks which are possible to be performed by young children but unlikely to be exhibited spontaneously were selected (Vanvuchelen et al. 2010). Non-imitative behaviour with the objects used in the PIPS was ruled out. The 10 action categories and 30 PIPS tasks are described in the "Appendix".

Imitation performances on each task are scored on a 3-5 point scale in accordance with the criteria of the scoring system of the PIPS, which evaluates the spatiotemporal resemblance between the modelled and copied action. To illustrate this system, we explain the scoring of the task "to pretend to comb your hair with an imaginary comb". Score 4 is given if the child has used a symbolic grip and has performed a repetitive action on both sides of the head. Score 3 is given if the child has used a symbolic grip and has performed a repetitive action on one side of the head or a single action. Score 2 is given if the child has used a body-part-asan-object grip and has performed a repetitive action on both sides of the head. Score 1 is given if the child has used a body-part-as-an-object grip and has performed a repetitive action on one side of the head or a single action. Score 0 is given is the child has performed another action or has refused to imitate (Vanvuchelen 2009). The final PIPS score is a reflection of the accuracy of the child's imitation performance. PIPS scale and subscale scores have high internal consistency. PIPS scores demonstrate acceptable intra- and interrater reliability. Results of test-retest analysis suggest that the PIPS score is stable over time. Bodily and procedural imitation age-equivalents (21, respectively 9 imitation tasks) as well as ageequivalent scores on the 15 meaningful goal directed and 15 non-meaningful non-goal directed tasks separately were derived from PIPS scores of 654 typically developing children between 12 and 59 months of age.

The participants in the present study were assessed by trained investigators of the University Clinics. An interrater agreement of the total score above $85 \%$ with the trainer (M.V.) was achieved by all investigators. Administration of the PIPS was in accordance with the guidelines for item instruction of the PIPS. Before administering the tasks of the PIPS, the child was given three introductory tasks. During these 3 tasks a broad range of instructions to evoke imitation was given to the child. The 30 tasks of the PIPS were presented in a 
standardised way. Before the demonstration of each action, the child's attention was attracted by calling her/his name. Only the verbal instruction "(Name), you do it too" was given. This instruction was given in Dutch. The time needed to complete the PIPS ranged from 10 to 20 min (Vanvuchelen 2009). Some team members were trained in the administration of both the PIPS and the ADOS. We could not prevent that a small number of children received the PIPS and the ADOS from the same team members. To avoid verification bias, we did not provide the normative data of the PIPS to the team members. As a consequence, they could not use the results on the imitation scale for their diagnostic decision-making.

\section{Measurements of Nonverbal Mental Level}

Since children suspected of autism may have language difficulties, nonverbal measures were used to assess the children's mental level. Standardised tests appropriate to the child's age were used: 27 participants (24 ASD and 3 NS-DD) were measured with the Dutch modification of the nonverbal version of mental scale of the Bayley Scales of Infant Development (BSID-II-NL; Van der Meulen et al. 2000) and 59 participants (44 ASD and 15 NS-DD) with the revised version of the Snijders-Oomen Nonverbal Intelligence Test for Children (SON-R 2.5-7; Tellegen et al. 1998). The mean time between imitation and mental assessment was 0.8 month (SD 1.9 month).

\section{Measurements of Receptive and Expressive Language Level}

Language reception level was measured using the MacArthur-Bates Communicative Development Inventories N-CDI "Words and Gestures" in 5 participants (5 ASD), N-CDI "Words and Sentences" in 10 participants (8 ASD and 2 NS-DD) (Zink and Lejaegere 2002) and Reynell Developmental Language Scales RTOS (Schaerlaekens et al. 2003) in 68 participants (53 ASD and 15 NS-DD). Language production level was measured using N-CDI "Words and Gestures" in 4 participants (4 ASD), N-CDI "Words and Sentences" in 13 participants (12 ASD and 1 NS-DD) and RTOS in 58 participants (44 ASD and 14 NS-DD). The mean time between imitation and language assessment was 0.73 month (SD 2.5 month).

\section{Measurement of Gross and Fine Motor Level}

Gross and fine motor level were measured in 75 participants (62 ASD and 12 NS-DD) with the use of the locomotor, respectively visuomotor integration subtest of the Peabody 
Developmental Motor Scales-2 (PDMS-2) (Folio and Fewell 2000). The mean time between imitation and motor assessment was 0.25 month (SD 0.9 month).

\section{Statistical Analysis}

For the analysis of the level of child participation, item scores were collapsed into two categories. Item score zero remains score zero (indicating no response) and item scores one till four were recoded to score one (indicating any attempt to imitate, regardless of the accuracy of the imitation performance). Group differences regarding the frequency of responses were checked with the Fisher-Exact test.

To take into account nonverbal mental age, all scores (i.e., imitation, language, motor) are expressed as a difference between age-equivalent scores and nonverbal mental age at the moment the specific tests are performed. As such, a participant having a score which corresponds with his/her nonverbal mental age will have a zero (difference) score. A negative score pertains to delay in relation to the participant's nonverbal mental age. A positive score pertains to advancement in relation to the participant's nonverbal mental age. In the result section, both the negative and positive difference scores are referred to as delay scores.

Differences between two groups regarding chronological age, nonverbal mental age and delay scores were checked with the Mann-Whitney $U$ test $(U)$. Differences between three subgroups of children with autism spectrum disorders (ASD) regarding chronological age were checked with the Kruskal-Wallis test. Within-group differences regarding nonverbal mental age and imitation, motor and language age equivalent scores were verified with a Wilcoxon signed ranks test $(Z)$.

Correlations between delay scores were examined with the Spearman correlation coefficient. Multiple linear regression analysis (stepwise) was used to explain the variance of the imitation delay scores.

Logistic regression models have been used to verify for each delay score separately the relation with the multidisciplinary team diagnosis. A Receiver Operating Characteristic (ROC) curve, plotting sensitivity against 1-specificity, is obtained using every observed data value to discriminate between both groups. In this way a concordance index (c-index) was achieved. This index is a quantification of the discriminatory performance of the delay score 
which corresponds to the area under the ROC curve. The c-index has also the meaning of a probability: the probability that when one takes a pair of participants, one with a positive and the other with a negative diagnosis, the one with the positive consensus diagnosis has a higher predicted probability to be positive. Thus, when the c-index equals 0.5 , random predictions are made. Finally, all six delay scores are combined into one multiple logistic regression model to assess the relative importance of each score. To verify the robustness of the obtained conclusions an alternative analysis has been performed. The logistic regression models are considered with the age-equivalent scores instead of the delay scores as predictor. Nonverbal mental age is then taken into account by using it as an additional covariate.

$p$-values smaller than 0.05 are considered as significant. All analyses have been performed using the statistical software SAS (version 9.1).

\section{Results}

\section{Level of Child Participation}

The two groups (ASD $n=68$ and NS-DD $n=18$ ) did not differ in frequency of response on 28 out of 30 tasks. Participants in the ASD group responded less frequently than participants in the NS-DD group on 2 tasks: item 5 and item 8 (Fisher Exact test, $p=0.04$, respectively $p=0.03$ ) (see "Appendix" for description of the items). Overall, these analyses indicated that the ASD group did not differ in cooperation during imitation assessment. Analyses of the maximum scores revealed that none of the participants achieved a maximum score on the bodily imitation tasks. Two ASD participants achieved a maximum score on the procedural imitation tasks. Overall, these analyses indicated that results were not confounded by ceiling effects.

\section{Preliminary Analysis of the Whole Sample $(n=86)$}

The two groups (ASD $n=68$ and NS-DD $n=18$ ) did not differ significantly in chronological age (CA, $U=463.0 ; p=0.11)$ and nonverbal mental age (NMA, $U=588.0 ; p=0.79$ ). 
For the 86 participants, there was clear evidence for nonverbal mental delay $(Z=3.99$; $p<0.001$ ). The median delay equals -3.9 months (Interquartile Range (IQR): $-10.8 ; 2.1$ ). Of all participants $69 \%(59 / 86)$ had a nonverbal mental age below their chronological age. This holds for 66\% (45/68) of the participants with ASD $(Z=2.98 ; p=0.003)$ and $78 \%(14 / 18)$ of these with NS-DD $(Z=3.11 ; p=0.002)$ (Fisher Exact test, $p=0.41)$. Therefore, analyses were conducted with delay scores in relation to nonverbal mental age (NMA).

Table 1 gives an overview of descriptive information for age (CA, NMA) and for the delay scores in relation to the nonverbal mental age for each of the considered measures: bodily (BID) and procedural (PID) imitation delay, receptive (RLD) and expressive (EDL) language delay, gross (GMD) and fine (FMD) motor delay. Figure 1 visualises the delay scores in both groups.

Table 1 Descriptive information of baseline characteristics and delay scores relative to nonverbal mental age expectancy (Median, Interquartile range) for children with a positive (ASD) and a negative (NS-DD) multidisciplinary team diagnosis separately

\begin{tabular}{|c|c|c|c|c|c|}
\hline & $\begin{array}{l}\text { ASD } \\
(n=68)\end{array}$ & $\begin{array}{l}\text { NS-DD } \\
(n=18)\end{array}$ & OR $(95 \% \mathrm{CI})$ & $\begin{array}{l}p \text { - } \\
\text { value }\end{array}$ & $\begin{array}{l}\text { C- } \\
\text { index }\end{array}$ \\
\hline $\begin{array}{l}\text { Chronological age (CA), } \\
\text { months }\end{array}$ & $\begin{array}{l}40.5 \\
(23 ; 48)\end{array}$ & $45.0(37 ; 52)$ & & 0.11 & \\
\hline $\begin{array}{l}\text { Nonverbal mental age (NMA), } \\
\text { months }\end{array}$ & $\begin{array}{l}36.0 \\
(27 ; 44)\end{array}$ & $35.6(31 ; 45)$ & & 0.79 & \\
\hline $\begin{array}{l}\text { Mental delay (NMA-CA), } \\
\text { months }\end{array}$ & $\begin{array}{l}-3.8 \\
(-11 ; 3)\end{array}$ & $-4.1(-13 ;-2)$ & & 0.28 & \\
\hline \multicolumn{6}{|l|}{ Delay scores (months) } \\
\hline Bodily imitation delay (BID) & $\begin{array}{l}-5.4 \\
(-12 ; 1)\end{array}$ & $-4.5(-8 ; 4)$ & $\begin{array}{l}1.021 \\
(0.969 ; 1.076)\end{array}$ & 0.43 & 0.573 \\
\hline $\begin{array}{l}\text { Procedural imitation delay } \\
\text { (PID) }\end{array}$ & $-3.1(-8 ; 5)$ & $4.7(-4 ; 12)$ & $\begin{array}{l}1.065 \\
(1.006 ; 1.126)\end{array}$ & 0.02 & 0.647 \\
\hline $\begin{array}{l}\text { Receptive language delay } \\
\text { (RLD) }\end{array}$ & $\begin{array}{l}-3.8 \\
(-10 ; 2)\end{array}$ & $-0.4(-5 ; 5)$ & $\begin{array}{l}1.076 \\
(0.998 ; 1.159)\end{array}$ & 0.03 & 0.637 \\
\hline $\begin{array}{l}\text { Expressive language delay } \\
\text { (ELD) }\end{array}$ & $\begin{array}{l}-3.9 \\
(-10 ; 1)\end{array}$ & $-3.8(-6 ; 1)$ & $\begin{array}{l}1.034 \\
(0.971 ; 1.102)\end{array}$ & 0.28 & 0.548 \\
\hline Gross motor delay (GMD) & $\begin{array}{l}-3.9 \\
(-11 ; 3)\end{array}$ & $0.0(-3 ; 2)$ & $\begin{array}{l}1.038 \\
(0.967 ; 1.113)\end{array}$ & 0.29 & 0.594 \\
\hline Fine motor delay (FMD) & $-1.8(-5 ; 3)$ & $1.3(-4 ; 4)$ & $\begin{array}{l}1.028 \\
(0.949 ; 1.113)\end{array}$ & 0.50 & 0.565 \\
\hline
\end{tabular}


Note that mental and chronological age (months) refer to the moment of PIPS, respectively language and motor assessment. Negative values for the delay scores refer to a delay with respect to nonverbal mental age. For continuous measurements, values are medians with the IQR between parentheses. For the delay scores, the odds ratio (OR, with 95\%CI between parentheses) and index of diagnostic performance (C-index) is given with a $p$-value obtained from the univariable logistic regression models. The odds ratio refers to the multiplicative effect of a one-unit decrease in score (hence, one unit 'more delay') on the odds for a positive diagnosis.
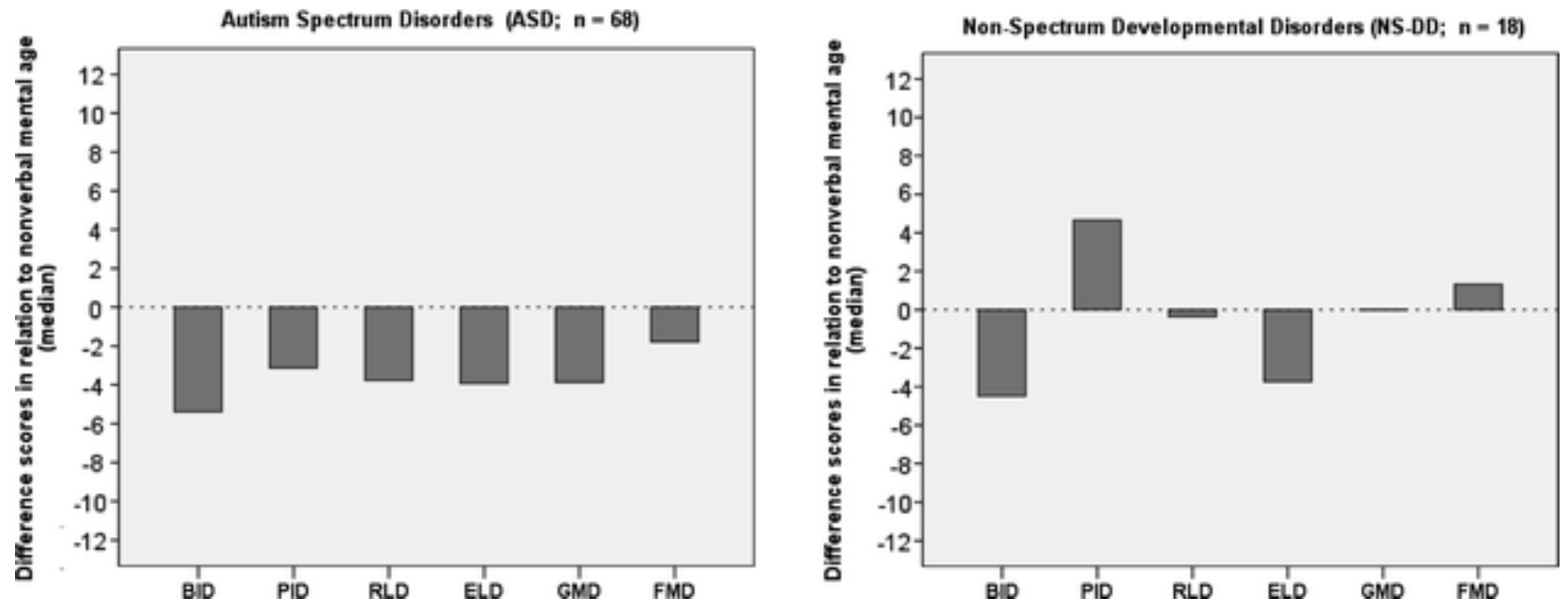

Fig. 1 Bar Charts of Bodily (BID) and Procedural Imitation Delay (PID), Receptive (RLD) and Expressive Language Delay (ELD), Gross (GMD) and Fine Motor Delay (FMD) Scores (i.e., differences between nonverbal mental age and age-equivalents scores) of children with autism spectrum disorders (ASD, $n=68$ ) and non-spectrum-developmental disorders (NS-DD, $n=18$ ). Note that a positive score pertains to advancement in relation to nonverbal mental age

In the total sample $(n=86)$, BID was significantly and positively correlated to the other delay scores. PID was significantly and positively correlated to motor delay scores and less strong to language delay scores (Table 2). Overall, these analyses indicate that imitation delay scores are clearly related to delays in other developmental domains. 
Table 2 Correlation matrix (Spearman rho) of delay scores for the whole sample $(n=86)$

\begin{tabular}{|l|l|l|l|l|l|l|}
\hline & BID & PID & RLD & ELD & GMD & FMD \\
\hline BID & - & & & & & \\
\hline PID & $0.42^{* *}$ & - & & & & \\
\hline RLD & $0.34 * *$ & 0.20 & - & & & \\
\hline ELD & $0.27 *$ & 0.21 & $0.58^{* *}$ & - & & \\
\hline GMD & $0.43^{* *}$ & $0.23^{*}$ & $0.55^{* *}$ & $0.42^{* *}$ & - & \\
\hline FMD & $0.47 * *$ & $0.30^{* *}$ & $0.47 * *$ & $0.45^{* *}$ & $0.66^{* *}$ & - \\
\hline
\end{tabular}

$B I D$ bodily imitation delay; PID procedural imitation delay, $R L D$ receptive language delay, $E L D$ expressive language delay, GMD gross motor delay, $F M D$ fine motor delay

* Correlation is significant at the 0.05 level (2-tailed), ${ }^{* *}$ Correlation is significant at the 0.01 level (2tailed)

Multiple linear regression analysis $(n=86)$ with the variables RLD, ELD, GMD, FMD and diagnoses (ASD vs. NS-DD) revealed one predictive factor for BID. Fine motor delay explained $24.5 \%$ of the variance of BID. The same analysis revealed two predictive factors for PID. Fine motor delay explained $18.2 \%$ and diagnoses an additional $12.8 \%$ of the variance of PID. These analyses indicate that bodily and procedural imitation delay can be partially explained by fine motor delay. In addition, procedural imitation delay can be partially explained by the diagnosis of ASD versus NS-DD.

\section{Are Imitation Problems Broad or Selective in Preschoolers with Autism?}

Within-group differences regarding imitation age and nonverbal mental age were verified. For the 68 participants with ASD, there was clear evidence for BID $(Z=4.04 ; p<0.001)$ and PID $(Z=2.12 ; p=0.03)$. There was no evidence for a difference between BID and PID $(Z=1.5$, $p=0.11)$.

To verify the finding of broad imitation problems a second analysis has been performed. The age-equivalent scores on the 15 meaningful goal directed and 15 non-meaningful non-goal directed imitation tasks were used. There was clear evidence for imitation delay in relation to 
the nonverbal mental age on the meaningful goal directed $(Z=3.24 ; p=0.001)$ and nonmeaningful non-goal directed $(Z=54.19 ; p<0.001)$ tasks. There was no evidence for a difference between meaningful goal directed and non-meaningful non-goal directed imitation delay $(Z=0.98, p=0.32)$.

Overall, these findings indicate broad imitation problems in ASD. In addition, there was clear evidence for $\operatorname{RLD}(Z=3.86 ; p<0.001)$, $\operatorname{ELD}(Z=3.43 ; p<0.001)$ and $\operatorname{GMD}(Z=3.13$; $p=0.002)$. Fine motor development was in proportion to the nonverbal mental age $(Z=1.58$; $p=0.11)$.

\section{Are Autism Imitation Problems Specific Instead of Being Part of Other Developmental Problems?}

Multiple linear regression analyses $(n=68)$ with the variables RLD, ELD, GMD, FMD were conducted. Results revealed just one predictive factor for BID and for PID. Fine motor delay explained $25.8 \%$ of the variance of BID and $18.3 \%$ of the variance of PID. These findings indicate that imitation delay in ASD can be partially explained by fine motor problems in relation to nonverbal mental age.

\section{Are Imitation Problems Universal in Autism?}

To address the question if imitation problems are universal in ASD, we divided participants with ASD in three subgroups according to the children's nonverbal mental abilities: 18 lowfunctioning (LFA: IQ < 80), 27 high-functioning (HFA: IQ $\geq 80$ and < 100) and 23 highestfunctioning (HHFA: IQ $\geq 100$ ) children. The three groups did not differ significantly in CA (Kruskal-Wallis Chi-Square $=0.12 ; p=0.93$ ).

Within-group differences regarding imitation age and nonverbal mental age were verified. In the 18 LFA participants, there was no evidence for imitation delay relative to the nonverbal mental age: $\operatorname{BID}(Z=0.11 ; p=0.90)$ and $\operatorname{PID}(Z=1.37 ; p=0.17)$. In addition, there was no 
evidence for $\operatorname{RLD}(Z=0.62 ; p=0.53), \operatorname{ELD}(Z=0.11 ; p=0.91), \operatorname{GMD}(Z=1.63 ; p=0.10)$ and FMD $(Z=1.72 ; p=0.08)$ (Fig. $\underline{2})$.
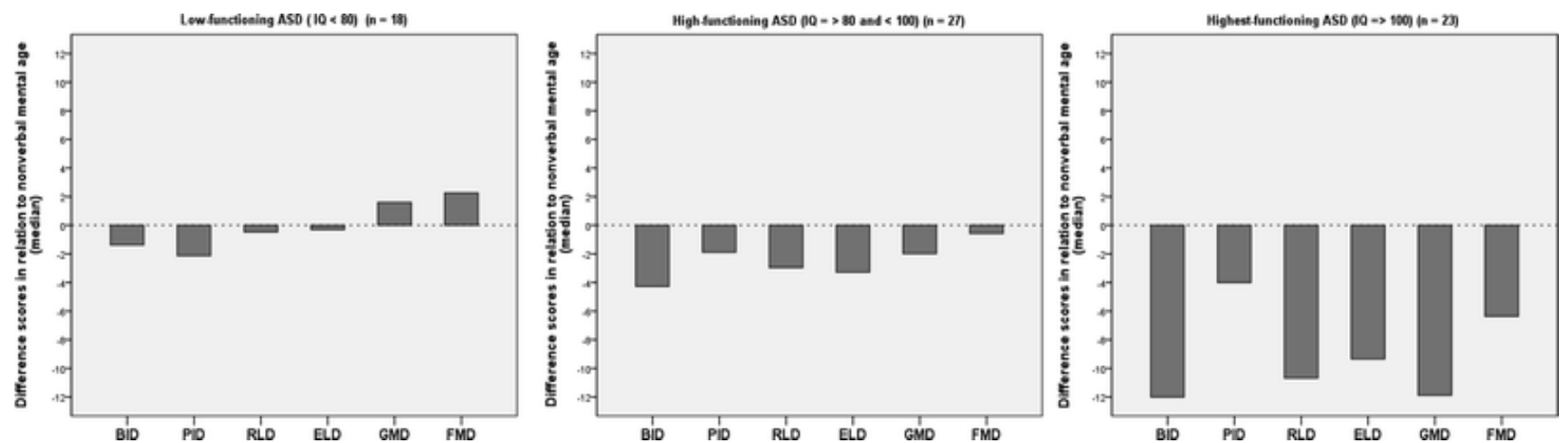

Fig. 2 Bar Charts of Bodily (BID) and Procedural Imitation Delay (PID), Receptive (RLD) and Expressive Language Delay (ELD), Gross (GMD) and Fine Motor Delay (FMD) Scores (i.e., differences between nonverbal mental age and age-equivalents scores) of children with lowfunctioning $(n=18)$, high-functioning $(n=27)$ and highest-functioning autism $(n=23)$

In the 27 HFA participants, there was evidence for bodily imitation delay (BID; $Z=2.28$; $p=0.02$ ), but not for procedural imitation delay (PID; $Z=0.19 ; p=0.84$ ) relative to the nonverbal mental age. In addition, there was some evidence for language delay relative to the nonverbal mental age: $\operatorname{ELD}(Z=1.99 ; p=0.04)$ and $\operatorname{RLD}(Z=1.77 ; p=0.07)$. There was no evidence for motor delay relative to the nonverbal mental age: $\operatorname{GMD}(Z=0.86 ; p=0.39)$ and $\operatorname{FMD}(Z=0.63 ; p=0.52)($ Fig. 2 ).

In the 23 HHFA participants with a nonverbal mental age above their CA, there was evidence for a delay relative to the nonverbal mental age in all developmental domains: $\operatorname{BID}(Z=3.95$; $p<0.001), \operatorname{PID}(Z=2.19 ; p=0.02), \operatorname{RLD}(Z=3.65 ; p<0.001), \operatorname{ELD}(Z=3.15 ; p=0.002)$, $\operatorname{GMD}(Z=4.07 ; p<0.001)$ and $\operatorname{FMD}(Z=3.19 ; p<0.001)$ (Fig. $\underline{2})$.

\section{Are Imitation Problems Unique to Autism at Preschool Age?}

Within-group differences in the NS-DD sample regarding imitation age and nonverbal mental age were verified. For the 18 participants with NS-DD, there was no evidence for imitation 
delay relative to the nonverbal mental age: $\operatorname{BID}(Z=1.50 ; p=0.13)$ and $\operatorname{PID}(Z=1.19$; $p=0.23)$. Procedural imitation was significantly better than bodily imitation $(Z=2.8$, $p=0.005)$. Note that bodily imitation age was below and procedural imitation above the children's nonverbal mental age (Table 1 and Fig. 1 ). In addition, there was no evidence for $\operatorname{RLD}(Z=0.02 ; p=0.98), \operatorname{ELD}(Z=1.47 ; p=0.14), \operatorname{GMD}(Z=0.54 ; p=0.58)$ and FMD $(Z=0.17 ; p=0.86)$. The NS-DD group was too small to be divided in subgroups according to the children's nonverbal mental abilities.

To address the question if imitation performance in NS-DD is specific or rather part of a delay in other developmental domains, multiple linear regression analyses with the variables RLD, ELD, GMD, FMD were conducted. Results revealed no predictive factors for BID and for PID. These findings indicate that imitation performance in NS-DD can not be explained by other developmental problems.

\section{What is the Diagnostic Utility of Imitation Assessment in Preschoolers Suspected of ASD?}

$\operatorname{ASD}(n=68)$ and NS-DD $(n=18)$ did not differ significantly in BID $(U=525.5 ; p=0.35)$. There was a trend that participants with ASD were more impaired in procedural imitation with respect to their nonverbal mental age than participants with NS-DD $(U=430.5$; $p=0.054)$. Both groups did not differ significantly in RLD $(U=406.5 ; p=0.08)$, ELD $(U=420.0 ; p=0.56), \operatorname{GMD}(U=328.0 ; p=0.29)$ and $\mathrm{FMD}(U=360.5 ; p=0.55)$.

In the sample of 86 referrals, there was evidence for a relation with the team diagnosis (ASD vs. NS-DD) for the procedural imitation delay $(p=0.021)$ and receptive language delay $(p=0.039)$ (logistic regression models, see Table $\underline{1})$. Figure $\underline{3}$ presents the ROC curve obtained from the univariable model for PIPS procedural imitation. Using the optimal cut-off for the delay score based on this curve yields a sensitivity of $82.4 \%$ (exact $95 \%$ confidence interval (CI):71.2; 90.5\%) and specificity of 50\% (CI: 26.0; 74.0\%). 


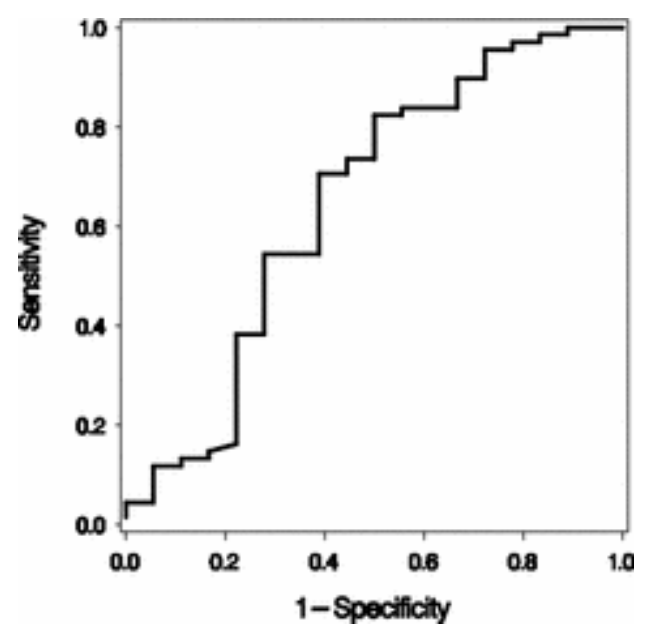

Fig. 3 Receiver Operating Characteristic (ROC) curve using the delay score for pips procedural imitation to discriminate between children with autism spectrum disorders (ASD, $n=68$ ) and nonspectrum-developmental disorders (NS-DD, $n=18$ ) Based on the multidisciplinary team diagnosis

A combination of the six delay scores in a multiple logistic regression model identified PIPS procedural imitation as the only significant predictor $(p=0.009)$ of diagnosis. The odds ratio for a change of 1 month towards more delay equalled 1.21 (CI: 1.05; 1.40). No evidence remained for a relation with receptive language level $(p=0.54)$.

The obtained conclusions were the same in the univariable as well as in the multivariable logistic regression models when using the age-corrected scores and nonverbal mental ages (at moment of test) as predictors instead of the delay scores (results not shown).

\section{Discussion}

This study is the first to investigate in a systematic way imitation aptitude of consecutively referred preschoolers suspected of autism. The main purpose was to address the question if imitation problems in preschoolers with autism met the criteria of a broad, specific, universal and unique deficit. If can be proved that imitation problems are a core characteristic in autism at preschool age, then the application of an age-specific imitation instrument may contribute to the early diagnosis of autism.

There are two basic methods to recruit participants for a diagnostic accuracy study. In a cohort type accuracy study a single set of inclusion criteria is used, in particular being suspected of having the disease. Participants are randomly selected and should represent the whole spectrum of severity level of the disease. In a case-control type accuracy study 
different sets of criteria are used to distinguish in advance patients with and without the target condition. Case-control type studies can be prone to bias. These studies compare the test results in cases with a clear disease with those in healthy 'controls' or 'controls' with other diseases. Since they use non-representative controls, findings of case-control study designs exaggerate the target problem (Jaeschke et al. 1994).

In the present study rigorous methodological procedures were used. First, we conducted a cohort type accuracy study with a random sample of preschoolers consecutively referred for suspected autism to four approved University Autism Clinics. This procedure should avoid the problem of selection bias (Lijmer et al. 1999). Second, we conducted the method of a direct head-to-head comparison (Lijmer et al. 1999). Autism features, imitation aptitude and developmental abilities were evaluated in the sample at the same time period. All participants received a clinical consensus diagnosis, an ADOS-G-classification (Lord et al. 2003), an imitation test and a mental ability assessment. This procedure avoided verification bias (Lijmer et al. 1999). This kind of bias could have loomed if the decision to perform the imitation test was based on the results of the clinical diagnosis or ADOS classification, or vice versa. Since the normative data of the imitation scale were not provided, the team members could not make use of these results in their diagnostic decision-making. In $73 \%$ of the participants the direct comparison was fully paired since these children received also language and motor ability assessments. Third, we used a standardised multidimensional age-specific imitation scale to assess core dimensions of imitation development in preschool children. The Preschool Imitation and Praxis Scale (PIPS) has sufficient reliability and validity to be used for clinical and research purposes (Vanvuchelen 2009; Vanvuchelen et al. 2010). The imitation age-equivalent scores of the PIPS offered the possibility to determine imitation delay in relation to the children's nonverbal mental age and to compare imitation delay directly with language and motor delay. Finally, prior to the analyses of the imitation performances, we have determined that group differences between the preschoolers with autism spectrum disorders (ASD) and with non-spectrum developmental disorders (NS-DD) were not due to a lack of responses on the part of either of the groups. This finding is consistent with results of Rogers et al. (2003). In addition, the use of age-specific imitation tasks has ruled out possible ceiling effects.

At group level, preschoolers with ASD showed broad imitation problems. They copy actions with salient environmental effects in procedural imitation tasks and action with internal 
effects in bodily imitation tasks below expectation, considering their nonverbal mental age. The same was true if the imitation tasks were divided with respect to the representational level of the actions, i.e., meaningful goal directed and non-meaningful non-goal directed. Results of brain imaging studies in healthy adults revealed two distinctive routes of imitation depending on the representational level of the actions. The imitation of actions of which the meaning or goal can only be identified retrospectively relies on a direct route, which transforms visuospatial characteristics into motor representations (Rumiati et al. 2005, 2009; Tessari and Rumiati 2004). Children may use this direct route of imitation to copy the non-meaningful bodily actions and the non-goal directed actions upon objects of the PIPS. They may faithfully copy the observable motor organisation of the demonstrator's act, i. e. the movement itself (the means) and the movement effect (the result). The imitation of familiar actions, for which the observer can identify a meaning or a goal and possesses a template in the long-term memory, relies on an indirect semantic-related route of imitation (Rumiati et al. 2005, 2009; Tessari and Rumiati 2004). Children may use this indirect route of imitation to copy the meaningful bodily actions and goal directed actions upon objects of the PIPS. They may copy the non-observable, inferable higher organisational structures of the demonstrators' actions, i.e., the mental representation of the demonstrator's desired end result (the goal) and the mental representation of the means the demonstrator has chosen to achieve the desired result (the intention). Findings of the present study revealed that there was no difference between the delay scores on both types of actions in any group. This finding suggests that possible underlying mechanisms of autism imitation problems involve both the direct and indirect route of imitation.

A next question that has been verified was whether or not autism imitation problems are specific. As hypothesised, results of multiple linear regression analyses revealed that in children with ASD bodily and procedural delays are predicted by the children's fine motor delay in relation to their nonverbal mental abilities. Therefore, we concluded that imitation problems are partially due to fine motor problems in ASD.

A critical conclusion of the present study is that imitation problems relative to the children's nonverbal mental age, are not universal in autism at preschool age. We failed to replicate findings of case-control studies in low-functioning children with autism that identified imitation problems at preschool age as a core characteristic in autism (for reviews see Rogers 1999; Smith and Bryson 1994; Williams et al. 2004). The use of delay scores relative to the 
children's nonverbal mental age in this study may explain the different findings. In the present study ASD children with an IQ below 80 did not show any imitation problem relative to their nonverbal mental ages. In both the autistic and non-autistic low-functioning children, the mental constraints seem to overshadow all other developmental domains, including imitation. Findings of the present study are partially consistent with the results of Rogers et al. s' study (2003). Rogers et al. (2003) found no gestural imitation problems in low-functioning toddlers with ASD compared to chronological and mental age matched non-autistic controls. But in their study, the children with autism showed facial and procedural imitation problems. Interesting is the finding of the present study that in autistic children with an IQ above 80 imitation problems became apparent. In these children bodily imitation was delayed in relation to their nonverbal mental age. And even more fascinating, was the finding that in children with ASD and an IQ above 100, both bodily and procedural imitation was significantly below their nonverbal mental ages. Thus, we found only adequate evidence for the idea that imitation problems are broad and specific with respect to mental abilities in the subgroup of highest-functioning preschoolers with ASD. To the best of our knowledge, there are no other studies which have investigated imitation aptitude in children with a mental age above their chronological age.

At first sight, there was some evidence that imitation problems are unique to autism. As hypothesised, imitation performance of preschoolers suspected of autism and who did not meet the criteria of autism was in accordance with their nonverbal mental abilities. However, results of between group analyses revealed only a trend that preschoolers with confirmed ASD were more impaired in procedural imitation than participants with NS-DD.

So the question remains: "What is the diagnostic utility of imitation assessment in preschoolers suspected of autism?" This is a captivating question, since the children in present study were 2 years younger than the average age of autism diagnosis in Flanders. Results of a predictive model using logistic regression analysis with the six delay factors we examined as a single factor revealed that only procedural imitation delay and receptive language delay were associated with the diagnosis of autism. Results of a multiple logistic regression model identified procedural imitation delay as the only significant predictor of autism. Sensitivity or the proportion of true positives that are correctly identified by the model, i.e., correctly diagnosed as ASD, is high (82\%). But, the proportion of true negatives that are correctly identified by the model (specificity), i.e., correctly diagnosed as NS-DD, is only $50 \%$. Since 
the research sample was a population of children suspected of autism and referred to specialised university clinics, one may expect that the characteristics of the NS-DD group may easily be confused with autistic behaviour. For that reason, one would expect a high rate of false positives.

Taken together, results of the present study suggest that imitation aptitude measured with the PIPS can not be used as diagnostic criterion in all children suspected of autism. The children's imitation level reflects their developmental state much the same as their mental, language and motor levels do. Low- and high-functioning children with autism and even more clearly children with autism with a nonverbal mental age above their chronological age seem to have a different imitation pattern. Results of the present study support evidence for a broad and specific imitation problem in the highest-functioning children with autism. As yet we cannot give a definite answer if this imitation delay is an autism diagnostic criterion in children with an IQ above 100. In our sample, the group of non-autistic children with an IQ above 100 was too small. The ability to provide better sample specification through additional ratings of agespecific symptom severity would allow a more personalised clinical diagnosis and treatment of young children with autism. Although it is too soon to include imitation assessment in diagnostic decision-making in preschool children suspected of autism, imitation assessment may well offer meaningful information. It may provide insight in the future learning capacity of the child. This is in contrast with most developmental tests, which investigate what the child achieved so far. In young children with autism, the pre-intervention level of imitation is an important predictive factor for the response to treatment and the developmental outcome (Rogers et al. 2006; Sallows and Graupner 2005).

Some critical remarks have to be made. The imitation delay in children with autism was partially explained by fine motor problems. But, only $91 \%$ of the children with autism received a fine motor evaluation. It might be that especially children who were suspected of having fine motor problems received this evaluation. For that reason, we have to be careful with the interpretation of the impact of fine motor aptitude on autism imitation problems. For practical reasons the imitation assessment was included in the diagnostic protocol used in the Autism Clinics. Some team members were trained in both the PIPS and the ADOS administration. We could not prevent that some children received the PIPS and the ADOS from the same members of the team. For that reason, there was not always a blind comparison between autism diagnosis and imitation aptitude. Although a cohort type accuracy study has 
many plus-points compared to case-control studies (Jaeschke et al. 1994; Lijmer et al. 1999), some difficulties have to be noted. Because of the predominance of cases diagnosed as ASD we may have underestimated the risk factors. Children with NS-DD were underrepresented in the present sample. This may have decreased the statistical power in our study. Furthermore, the severity of this comparison groups' social and communication deficits as a result of having been suspected of ASD, may have contributed to the relative poor specificity of the PIPS. These children may be expected to produce false-positive results more often than otherwise healthy children or clearly non-autistic developmentally delayed children. Finally, there was considerable variability in imitation performances within the ASD group. According to longitudinal and retrospective studies not all children with autism clearly exhibit abnormalities early in life. Up to 50\% may first display a more or less typical development followed by loss of social and communication skills (Landa et al. 2007; Luyster et al. 2005).

\section{Conclusion}

Accrued findings of this study suggest that the developmental pattern, including imitation development of autistic preschoolers is very different depending on the children's mental capacity. In the sample of mentally impaired children with autism, imitation problems were obscured by the mental impairment. The higher the intelligence of the child with autism, the more apparent it became that imitation problems met the criterion of broadness and specificity in relation to mental abilities. However, imitation problems at preschool age were also partially explained by fine motor problems. Furthermore, findings of this study suggest that delay in procedural imitation that goes beyond the nonverbal mental delay may predict the diagnosis of autism. These results should be interpreted with caution until the study is replicated in other research samples, including a group of children suspected of having autism without mental delay. There is also a need for replication in other recruitment settings, which may allow involving other clinical groups.

Acknowledgments This study was supported by a Foundation Marguerite-Marie Delacroix grant (Tienen, Belgium) to M. Vanvuchelen. We would also like to thank all children, parents and staff of the University Clinics of Antwerpen, Brussel, Gent and Leuven (Belgium) who participated in this research. We thank Dr. S. Fieuws of L-BioStat (K.U.Leuven) for assistance with data analyses. This study was part of the doctoral dissertation of $\mathrm{M}$. Vanvuchelen. 


\section{Appendix}

Table 3 Description of the 30 Items of the Preschool Imitation and Praxis Scale (PIPS) presented in standardised order

\begin{tabular}{|c|c|c|c|c|}
\hline $\begin{array}{l}\text { Item } \\
\text { nr }\end{array}$ & $\mathbf{T}$ & Category & Dimension & Task description \\
\hline PIPS1 & $\mathrm{P}$ & sao-P1 & GDP & Raise a toy bear by pulling a cord \\
\hline PIPS2 & $\mathrm{P}$ & sao-P2 & GDP & Put a wooden block on top of your head \\
\hline PIPS3 & $\mathrm{P}$ & sao-P3 & GDP & Switch on a lamp in a toy animal with your forehead \\
\hline PIPS4 & $\mathrm{G}$ & i-MG1 & SIB & Perform the gesture to "wave good-bye" \\
\hline PIPS5 & G & i-MG2 & SIB & $\begin{array}{l}\text { Perform the gesture to "show something with an } \\
\text { outstretched hand in supination" }\end{array}$ \\
\hline PIPS6 & $\mathrm{G}$ & i-MG3 & SIB & Perform the gesture to "beckon with the index finger" \\
\hline PIPS7 & G & si-NMG1 & SIB & $\begin{array}{l}\text { Raise your outstretched arm till } 90^{\circ} \text { anteflexion and make a } \\
\text { circle with the index finger and thumb }\end{array}$ \\
\hline PIPS8 & G & si-NMG2 & SIB & $\begin{array}{l}\text { Raise your outstretched arm till } 90^{\circ} \text { anteflexion and stretch } \\
\text { out your fingers }\end{array}$ \\
\hline PIPS9 & G & si-NMG3 & SIB & $\begin{array}{l}\text { Raise your outstretched arm till } 90^{\circ} \text { anteflexion, hold up } \\
\text { the little finger while all the other fingers and the thumb are } \\
\text { bent }\end{array}$ \\
\hline PIPS10 & $\mathrm{P}$ & sq-P1 & NGDP & $\begin{array}{l}\text { Open the box, put the lid on the table, turn the box upside- } \\
\text { down, put the block on the bottom of the box }\end{array}$ \\
\hline PIPS11 & $\mathrm{P}$ & sq-P2 & NGDP & $\begin{array}{l}\text { Take the block from the bottom of the box, turn the box in } \\
\text { normal position again, close the box, put the block on the } \\
\text { lid of the box }\end{array}$ \\
\hline PIPS12 & $\mathrm{P}$ & sq-P3 & NGDP & $\begin{array}{l}\text { Take the block from the lid of the box, open the box, put a } \\
\text { disc into the box, close the box, put the block again on the } \\
\text { lid of the box }\end{array}$ \\
\hline PIPS13 & $\mathrm{G}$ & $\mathrm{t}-\mathrm{MG} 1$ & SIB & Pretend to "comb your hair with an imaginary comb" \\
\hline PIPS14 & G & t-MG2 & SIB & $\begin{array}{l}\text { Pretend to "open an imaginary door with an imaginary } \\
\text { key" }\end{array}$ \\
\hline PIPS15 & $\mathrm{G}$ & t-MG3 & SIB & Pretend to "brush your teeth with an imaginary toothbrush" \\
\hline PIPS16 & $\mathrm{G}$ & bi-NMG1 & SIB & Place one fist on top of the other \\
\hline PIPS17 & G & bi-NMG2 & SIB & $\begin{array}{l}\text { Extend the index fingers of both hands while the other } \\
\text { fingers and thumbs are bent, and bring the top of the index } \\
\text { fingers towards each other }\end{array}$ \\
\hline PIPS18 & $\mathrm{G}$ & bi-NMG3 & SIB & $\begin{array}{l}\text { Open one hand in vertical position and touch the top of the } \\
\text { fingers with the palm of the other hand in horizontal } \\
\text { position }\end{array}$ \\
\hline PIPS19 & $\mathrm{G}$ & fa-NMG1 & SIB & Extend your index finger and touch the top of your nose \\
\hline
\end{tabular}




\begin{tabular}{|c|c|c|c|c|}
\hline $\begin{array}{l}\text { Item } \\
\text { nr }\end{array}$ & $\mathbf{T}$ & Category & Dimension & Task description \\
\hline PIPS20 & $\mathrm{G}$ & fa-NMG2 & SIB & Touch your lower lips with the nails of your thumbs \\
\hline PIPS21 & $\mathrm{G}$ & fa-NMG3 & SIB & $\begin{array}{l}\text { Extend the index finger of your left hand and touch your } \\
\text { right cheek and extend the index finger of your right hand } \\
\text { and touch your left cheek }\end{array}$ \\
\hline PIPS22 & $\mathrm{P}$ & aso-P1 & GDP & $\begin{array}{l}\text { Turn a cup upside-down and play drums on it with two } \\
\text { spoons }\end{array}$ \\
\hline PIPS23 & $\mathrm{P}$ & aso-P2 & GDP & $\begin{array}{l}\text { Remove the cap of a doll and put a shoe on the head of the } \\
\text { doll }\end{array}$ \\
\hline PIPS24 & $\mathrm{P}$ & aso-P3 & GDP & $\begin{array}{l}\text { Put a toy car in bed, turn it upside-down and tuck it in with } \\
\text { a blanket }\end{array}$ \\
\hline PIPS25 & G & $\begin{array}{l}\text { sq- } \\
\text { NMG1 }\end{array}$ & SQB & $\begin{array}{l}\text { Hit the table with the palm of your hands, cross the arms } \\
\text { and hit the table again, return to the original position and } \\
\text { hit the table once more }\end{array}$ \\
\hline PIPS26 & $\mathrm{G}$ & $\begin{array}{l}\text { sq- } \\
\text { NMG2 }\end{array}$ & SQB & $\begin{array}{l}\text { Hit the table with one hand in supination, turn the hand in } \\
\text { pronation and hit the table again, clap in the hands, hit the } \\
\text { table with the palm of both hands }\end{array}$ \\
\hline PIPS27 & $\mathrm{G}$ & $\begin{array}{l}\text { sq- } \\
\text { NMG3 }\end{array}$ & SQB & $\begin{array}{l}\text { Hit the table with both hands in supination, turn the hands } \\
\text { in pronation, hit the table again, clap in the hands, hit the } \\
\text { table with the palm of both hands once more }\end{array}$ \\
\hline PIPS28 & $\mathrm{F}$ & $\mathrm{F} 1$ & SIB & $\begin{array}{l}\text { Shake the head, eyes closed to say 'no', with an expression } \\
\text { of disapproval }\end{array}$ \\
\hline PIPS29 & $\mathrm{F}$ & $\mathrm{F} 2$ & SIB & Look angry with a frown of the eyebrows \\
\hline PIPS30 & $\mathrm{F}$ & F3 & SIB & $\begin{array}{l}\text { Nod quickly with your head and show an expression of } \\
\text { happiness }\end{array}$ \\
\hline
\end{tabular}

$T$ Type of action, $P$ procedural imitation, $G$ gestural imitation, $F$ facial imitation, sao- $P$ substitutedactions-upon-objects, $i-M G$ intransitive meaningful gestures, si-NMG single non-meaningful hand postures, $s q-P$ action-sequences-upon-objects, $t-M G$ transitive meaningful gestures, bi-NMG bimanual non-meaningful hand postures, fa-NMG non-meaningful hand postures to the face or head, aso- $P$ actions-upon-substituted-objects, sq-NMG sequences of non-meaningful hand postures, $f$ facial expressions, GDP goal directed procedural imitation, NGDP non-goal directed procedural imitation, $S I B$ single bodily imitation, $S Q B$ sequential bodily imitation 


\section{References}

Aldridge, M. A., Stone, K. R., Sweeney, M. H., \& Bower, T. G. R. (2000). Preverbal children with autism understand the intentions of others. Developmental Science, 3, 294-301.

American Psychiatric Association. (2000). Diagnostic and statistical manual of mental disorders DSM-IV-TR (Text Revison). Washington DC: American Psychiatric Association.

Beadle-Brown, J., \& Whiten, A. (2004). Elicited imitation in children and adults with autism: Is there a deficit? Journal of Intellectual \& Developmental Disability, 29, 147-163.

Carpenter, M., Pennington, B. F., \& Rogers, S. J. (2002). Interrelations among socialcognitive skills in young children with autism. Journal of Autism and Developmental Disorders, 32, 91-106.

Charman, T., \& Baird, G. (2002). Practitioner review: Diagnosis of autism spectrum disorder in 2- and 3-year-old children. Journal of Child Psychology and Psychiatry, 43, 289-305.

Charman, T., Swettenham, J., Baron-Cohen, S., Cox, A., Baird, G., \& Drew, A. (1997). Infants with autism: An investigation of empathy, pretend play, joint attention, and imitation. Developmental Psychology, 33, 781-789.

Chawarska, K., Klin, A., Paul, R., \& Volkmar, F. (2007). Autism spectrum disorder in the second year: Stability and change in syndrome expression. Journal of Child Psychology and Psychiatry, 48, 128-138.

DeMeyer, M. K., Alpern, G. D., Barton, S., DeMyer, W. E., Churchill, D. W., Hingtgen, J. N., et al. (1972). Imitation in autistic, early schizophrenic, and non-psychotic subnormal children. Journal of Autism and Childhood Schizophrenia, 2, 264-287.

Folio, M. R., \& Fewell, R. R. (2000). Peabody Developmental Motor Scales. Guide to item Administration (2nd ed.). Austin Texas: Pro-Ed.

Hobson, R. P., \& Lee, A. (1999). Imitation and identification in autism. Journal of Child Psychology and Psychiatry, 40, 649-659.

Ingersoll, B., Schreibman, L., \& Tran, Q. H. (2003). Effect of sensory feedback on immediate object imitation in children with autism. Journal of Autism and Developmental Disorders, 33, $673-683$. 
Jaeschke, R., Guyatt, G., \& Sackett, D. L. (1994). Users' guides to the medical literature. III. How to use an article about a diagnostic test. A. Are the results of the study valid? EvidenceBased Medicine Working Group. JAMA: The Journal of the American Medical Association, 271, 389-391.

Kleinman, J. M., Ventola, P. E., Pandey, J., Verbalis, A. D., Barton, M., Hodgson, S., et al. (2008). Diagnostic stability in very young children with autism spectrum disorders. Journal of Autism and Developmental Disorders, 38, 606-615.

Landa, R. J., Holman, K. C., \& Garrett-Mayer, E. (2007). Social and communication development in toddlers with early and later diagnosis of autism spectrum disorders. Archives of General Psychiatry, 64, 853-864.

Lijmer, J. G., Mol, B. W., Heisterkamp, S., Bonsel, G. J., Prins, M. H., van der Meulen, J. H., et al. (1999). Empirical evidence of design-related bias in studies of diagnostic tests. JAMA: The Journal of the American Medical Association, 282, 1061-1066.

Lord, C., Rutter, M., DiLavore, P. C., \& Risi, S. (2003). Autism diagnostic observation schedule. Manual. Los Angeles, CA: Western Psychological Services.

Luyster, R., Richler, J., Risi, S., Hsu, W. L., Dawson, G., Bernier, R., et al. (2005). Early regression in social communication in autism spectrum disorders: A CPEA study. Developmental Neuropsychology, 27, 311-336.

McDonough, L., Stahmer, A., Schreibman, L., \& Thompson, S. J. (1997). Deficits, delays, and distractions: An evaluation of symbolic play and memory in children with autism. Development and Psychopathology, 9, 17-41.

Roeyers, H. (2008). Autisme: Alles op een rijtje. Leuven: Acco.

Roeyers, H., Van Oost, P., \& Bothuyne, S. (1998). Immediate imitation and joint attention in young children with autism. Development and Psychopathology, 10, 441-450.

Rogers, S. J. (1999). An examination of the imitation deficit in autism. In J. Nadel \& G. Butterworth (Eds.), Imitation in infancy (pp. 254-283). Cambridge: Cambridge University Press.

Rogers, S. J., Hayden, D., Hepburn, S., Charlifue-Smith, R., Hall, T., \& Hayes, A. (2006). Teaching young nonverbal children with autism useful speech: A pilot study of the Denver 
Model and PROMPT interventions. Journal of Autism and Developmental Disorders, 36, $1007-1024$.

Rogers, S. J., Hepburn, S. L., Stackhouse, T., \& Wehner, E. (2003). Imitation performance in toddlers with autism and those with other developmental disorders. Journal of Child Psychology and Psychiatry, 44, 763-781.

Rogers, S. J., \& Williams, J. H. G. (2006). Imitation in Autism. Findings and Contraversies. In S. J. Rogers \& J. H. G. Williams (Eds.), Imitation and the social mind. Autism and typical development (pp. 277-309). New York: The Guilford Press.

Rogers, S. J., Young, G. S., Cook, I., Giolzetti, A., \& Ozonoff, S. 2008). Deferred and immediate imitation in regressive and early onset autism. Journal of Child Psychology and Psychiatry, 49, 449-457.

Rumiati, R. I., Carmo, J. C., \& Corradi-Dell'Acqua, C. (2009). Neuropsychological perspectives on the mechanisms of imitation. Philosophical Transactions of the Royal Society. Series B, Biological Sciences, 364, 2337-2347.

Rumiati, R. I., Weiss, P. H., Tessari, A., Assmus, A., Zilles, K., Herzog, H., et al. (2005). Common and differential neural mechanisms supporting imitation of meaningful and meaningless actions. Journal of Cognitive Neuroscience, 17, 1420-1431.

Sallows, G. O.,\&Graupner, T.D. (2005). Intensive behavioral treatment for children with autism: Four-year outcome and predictors. American Journal of Mental Retardation, 110, $417-438$.

Schaerlaekens, A., Zink, I., \& Van Ommeslaeghe, K. (2003). Reynell Taalontwikkelingsschalen. RTOS. Handleiding. Lisse: Swets Test Publishers.

Shattuck, P. T., Durkin, M., Maenner, M., Newschaffer, C., Mandell, D. S., Wiggins, L., et al. (2009). Timing of identification among children with an autism spectrum disorder: Findings from a population-based surveillance study. Journal of the American Academy of Child and Adolescent Psychiatry, 48(5), 474-483.

Sigman, M., Dijamco, A., Gratier, M., \& Rozga, A. (2004). Early detection of core deficits in autism. Mental Retardation and Developmental Disabilities Research Reviews, 10, 221-233. 
Smith, I. M., \& Bryson, S. E. (1994). Imitation and action in autism: A critical review. Psychological Bulletin, 116, 259-273.

Southgate, V., \& Hamilton, A. F. (2008). Unbroken mirrors: Challenging a theory of Autism. Trends in Cognitive Sciences, 12, 225-229.

Stone, W. L., Lemanek, K. L., Fishel, P. T., Fernandez, M. C., \& Altemeier, W. A. (1990). Play and imitation skills in the diagnosis of autism in young children. Pediatrics, 86, 267-272.

Stone, W. L., Ousley, O. Y., \& Littleford, C. D. (1997). Motor imitation in young children with autism: What's the object? Journal of Abnormal Child Psychology, 25, 475-485.

Tellegen, P., Winkel, M., Wijnberg-Williams, B., \& Laros, J. (1998). Snijders-Oomen Nietverbale Intelligentietest. SON-R 2_-7. Handleiding en Verantwoording. Lisse: Swets Test Publishers.

Tessari, A., \& Rumiati, R. I. (2004). The strategic control of multiple routes in imitation of actions. Journal of Experimental Psychology: Human Perception and Performance, 30, 11071116.

Van der Meulen, B. F., Ruiter, S. A. J., Lutje Spelberg, H. C., \& Smrkovsky, M. (2000). Bayley scales of infant development. Nederlandse versie. BSID-II-NL. Lisse: Swets Test Publishers.

Vanvuchelen, M. (2009). Preschool Imitation and Praxis Scale (PIPS). In: M. Vanvuchelen (ed.), Imitation problems in children with autism spectrum disorders. A study of their nature, clinical significance and utility in diagnosis, Ph D Dissertation in Rehabilitation Sciences and Physiotherapy, Group of Biomedical Sciences, Katholieke Universiteit Leuven (Belgium).

Vanvuchelen, M., Roeyers, H., \& De Weerdt, W. (2010). Development and initial validation of the Preschool Imitation and Praxis Scale (PIPS). Research in Autism Spectrum Disorders, doi: 10.1016/j.rasd.2010.06.010.

Wiggins, L. D., Baio, J., \& Rice, C. (2006). Examination of the time between first evaluation and first autism spectrum diagnosis in a population-based sample. Journal of Developmental and Behavioral Pediatrics, 27, S79-S87.

Williams, J. H., Whiten, A., \& Singh, T. (2004). A systematic review of action imitation in autistic spectrum disorder. Journal of Autism and Developmental Disorders, 34, 285-299. 
Zink, I., \& Lejaegere, M. (2002). N-CDIs. Lijsten voor Communicatieve Ontwikkeling. Aanpassing en hernormering van de MacArthur CDIs van Fenson et al.. Leuven: Acco.

Zwaigenbaum, L., Bryson, S., Rogers, T., Roberts, W., Brian, J., \& Szatmari, P. (2005). Behavioral manifestations of autism in the first year of life. International Journal of Developmental Neuroscience, 23, 143-152. 